\title{
La política cultural en territorio: análisis de Puntos de Cultura desde la dimensión del reconocimiento
}

\author{
Cultural policy on territory: Puntos de Cultura from \\ the dimension of recognition
}

\section{Romina Sánchez-Salinas}

IMESC-IDEHESI-CONICET, Universidad Nacional de Cuyo, Mendoza/ Universidad Nacional de Tres de Febrero, Buenos Aires, Argentina

rssalinasduntref.edu.ar

http://orcid.org/0000-0001-5269-900X

\section{Clarisa Inés Fernández}

Instituto de Investigaciones en Humanidades y

Ciencias Sociales (CONICET-UNLP), La Plata, Argentina

clarisafernandezaconicet.gov.ar

http://orcid.org/0000-0001-6760-1968

\section{Resumen}

El artículo analiza la implementación del Programa Puntos de Cultura en Argentina en su dimensión institucional y territorial observando dos organizaciones culturales comunitarias: el caso de Chacras para Todos (Mendoza) y el caso de la cooperativa La Comunitaria (Buenos Aires-La Pampa). La metodología utilizada ha sido cualitativa, valiéndonos de entrevistas en profundidad, observación participante y análisis de fuentes secundarias en el período 2013-2018. Los principales resultados indican que se trata de una política de alto impacto en la dimensión institucional de las organizaciones, volviendo legítimo el accionar de las mismas en sus territorios. A su vez, los procesos son impulsados y mediados principalmente por los proyectos y dinámicas de las organizaciones. Finalmente, la indagación demuestra que, cuando se incorporan al análisis las distintas escalas (local, nacional e internacional), el Programa presenta un alto impacto como política de reconocimiento.

Palabras clave: políticas culturales, organizaciones comunitarias, reconocimiento, Puntos de Cultura, Argentina.

\begin{abstract}
The article analyzes the performance of the policy Puntos de Cultura in Argentina regarding both its institutional and community dimensions. To do so, two local cultural organizations were observed as case studies: Chacras para Todos (Mendoza) and the co-op La Comunitaria (Buenos Aires-La Pampa). We applied a qualitative design, conducting in-depth interviews, participant observation, and reviewing secondary sources over the 2013-2018 period. The main results indicate that this is a highimpact policy in the institutional dimension of the organizations, legitimating their actions in their communities. The results also point out that the processes in the community dimension are mainly driven and mediated by the organizations' projects and dynamics. Finally, the study shows that the policy has a strong impact when incorporating different scales (local, national and international) into the analysis.
\end{abstract}

Keywords: cultural policies; community organizations; recognition; Puntos de Cultura; Argentina. 


\section{Introducción}

El artículo analiza la política de Puntos de Cultura $(\mathrm{PdC})$, iniciativa que ha adquirido notable relevancia en los debates y acciones sobre políticas culturales en Latinoamérica durante las primeras décadas del siglo XXI. Nos centraremos en revisar críticamente su implementación en Argentina, observando dos organizaciones comunitarias en el período 20132018. Los objetivos que guían la reflexión del artículo se vinculan a los procesos que se desarrollan en las organizaciones y sus comunidades a partir de la implementación del Programa y las dimensiones en las que éste impacta. Desde una perspectiva que integra las distintas escalas en juego, observamos cómo una política nacional que sustenta su legitimidad en redes y programas internacionales es, al mismo tiempo, apropiada y resignificada por las organizaciones locales a través de usos diferenciales de "repertorios de clasificación y nominación a los que recurren para construir definiciones colectivas" (Noel, 2011, p. 39).

Reconocemos, junto con Barbieri, Partal y Merino (2011), que es fundamental repensar diversas metodologías de evaluación de las políticas culturales públicas, que no estén centradas en relevar solo aspectos de las "externalidades de la cultura" (p.24), o circunscritas a registros cuantitativos o de consumo. Es, en ese sentido, que recuperamos la idea de construir un análisis que registre simultáneamente aspectos relativos a la intervención territorial de la política en su dimensión más visible y material, así como también aquellos procesos intangibles que operan en el orden de lo simbólico y la experiencia de quienes integran los grupos involucrados. A partir de estas premisas, identificamos un área aún poco explorada en la evaluación de políticas culturales de base comunitaria, a la que esperamos realizar un aporte con este trabajo. Asimismo, la revisión de estudios previos sobre PdC en Argentina indica que la mayor parte abordan el Programa en tanto política pública: sus inicios, los paradigmas en los que se inscribe, los conceptos de cultura que promueve, el análisis de su impacto, entre otros ${ }^{1}$. En este trabajo posamos la mirada sobre las organizaciones, es decir, ahondamos en los significados y dinámicas que se despliegan en las comunidades a partir de la intervención de la política.
Las reflexiones se basan en investigaciones previas de las autoras de este trabajo, donde encontramos un denominador común: el teatro comunitario argentino ${ }^{2}$ como objeto de estudio (Fernández, 2015, 2018, 2020; Fernández et al., 2017; Sánchez Salinas, 2014, 2018a, 2018b, 2020). Respecto a las perspectivas teóricas utilizadas, pondremos en diálogo herramientas de distintas disciplinas como la sociología, la antropología, la gestión cultural y los estudios culturales.

Trabajamos con una metodología cualitativa, valiéndonos de entrevistas en profundidad ${ }^{3}$, observación participante y consulta de fuentes secundarias (Marradi, Archenti \& Piovani, 2007). El recorte temporal responde a los momentos claves de articulación entre las organizaciones y el PdC (2013-2018), pero también incluye procesos que se extienden a la actualidad. Los datos extraídos del trabajo de campo son analizados en clave contextual desde una perspectiva histórica y relacional, procurando evitar miradas duales al momento de analizar la relación entre el Estado y las organizaciones de la sociedad civil.

Los casos seleccionados son la Cooperativa La Comunitaria (provincias de Buenos Aires y La Pampa) y Chacras para todos (provincia de Mendoza). Ambos casos presentan características comunes que los vuelven significativos para el análisis del problema. En primer lugar, se trata de organizaciones que, en sus territorios, son referentes de la cultura comunitaria, no sólo a nivel local, sino también porque representan a organizaciones de la región en las redes culturales del sector. Ambos proyectos comenzaron como grupos de teatro comunitario, integran la Red Nacional de Teatro Comunitario"y participan activamente en el Movimiento de Cultura Viva Comunitaria (CVC). Además, se ubican en pueblos o ciudades intermedias alejadas de los centros donde se diseñan y disputan los sentidos de las políticas culturales. Esto es particularmente relevante dentro de los grupos de teatro comunitario, ya que presentan, en general, una tradición muy acentuada en la Ciudad Autónoma de Buenos Aires. Los estudios y experiencias de grupos de otras provincias tienen menor visibilidad y son escasos (Sánchez Salinas et al., 2014; Fernández et al., 2017). Por último, se trata de organizaciones comunitarias con cierta estabilidad, antigüedad e 
institucionalidad (más de 10 años) que se han vinculado en distintas ocasiones con PdC a través de la financiación de eventos y capacitaciones, entre otros.

El artículo, describe, en primer lugar, la política de PdC, para luego avanzar con el análisis de los casos en su articulación con el Programa y, finalmente, desarrolla una serie de reflexiones que configurarán nuevos aportes para pensar la relación entre Estado y organizaciones comunitarias, específicamente aquellas pertenecientes al campo del arte y la cultura.

\section{El Programa Puntos de Cultura en Argentina}

El programa nacional PdC fue presentado oficialmente en Argentina en el año 2011, a través de la Dirección Nacional de Participación y Organización Popular de la Secretaría de Políticas Socioculturales del Ministerio de Cultura de la Nación (Secretaría de Cultura de la Nación, 2011). El mismo brinda apoyo económico, técnico y capacitaciones a redes de cultura comunitaria y organizaciones de la sociedad civil que desarrollen proyectos culturales comunitarios para la inclusión social, la revalorización de la identidad local y la participación ciudadana (Benhabib, 2018).

El lanzamiento del Programa en el país se produjo gracias a una conjunción de procesos que venían dándose a nivel internacional, regional y nacional. A nivel internacional encontramos un importante movimiento de organizaciones y organismos a inicios del siglo XXI, que destacan la relevancia de la cultura como factor imprescindible para el desarrollo de las sociedades, a partir de la mirada de la diversidad y el rescate de la identidad (Organización de las Naciones Unidas para la Educación, la Ciencia y la Cultura, 2001, 2005). A nivel regional, en Brasil, la gestión presidencial de Luiz Inácio Lula da Silva y su ministro de cultura, Gilberto Gil, creó en el año 2004 el Programa Cultura Viva (PCV) de Cultura, Educación y Ciudadanía. Dentro del PCV encontramos el antecedente de Pontos de Cultura, que brindaba ayuda económica a organizaciones comunitarias de la sociedad civil y las integraba en una red de intercambio de experiencias, fortale- ciendo a aquellas organizaciones que contaran con una trayectoria comprobable en trabajo territorial y privilegiando las experiencias de contextos con mayor vulnerabilidad social.

En paralelo, diversas redes latinoamericanas y caribeñas - como la Red de Latinoamericana de Arte y Transformación Social o la Articulación Latinoamericana de Cultura y Política- se nuclearon en la Plataforma Puente Cultura Viva Comunitaria, la cual proponía que se destine el $0.1 \%$ de los presupuestos nacionales a la actividad cultural comunitaria, independiente y autogestiva. En Argentina, este movimiento tuvo su organización representante en el colectivo Pueblo Hace Cultura, conformado por organizaciones sociales, culturales, medios de comunicación populares y fuerzas políticas de todo el país.

PdC ha sido objeto de reflexión por parte de los propios funcionarios que estuvieron involucrados en su surgimiento e implementación, tanto en Brasil (Turino, 2013; Santini, 2017) como en Argentina (Benhabib, 2018; FuentesFirmani, s/f; Lucesole, 2016). Otras investigaciones lo han abordado como objeto de análisis (Mihal, 2014; Lacarrieu \& Cerdeira, 2016; Rodrigues de Melo, 2016; Brizuela \& Barrios, 2017; Wortman, 2017; Mello, 2018, 2019) o estudiaron su implementación e impacto en Brasil (Barbosa \& Calabre, 2011; Vilutis, 2009; Bentes, 2016; Rachild, 2016) y en Argentina (Prato \& Segura, 2018; Fernández, 2020; Sánchez Salinas, 2020; Wajnerman, 2018; Borda-Chauvin, 2020), por mencionar sólo algunos.

Benhabib (2018) postula tres puntos importantes que constituyen la política de PdC: la transmisión directa de fondos hacia las instituciones a través de las distintas líneas de aplicabilidad del programa, el fortalecimiento de una Red Nacional de PdC - a partir del armado de una Comisión Nacional de $\mathrm{PdC}$ y un Consejo Cultural Comunitario ${ }^{5}$ que permita construir una co-gestión participativa- y la formación e intercambio de experiencias. Con variaciones importantes, este Programa se ha mantenido a lo largo de los años, hasta constituirse en un modelo a seguir por varios países latinoamericanos. Por otro lado:

El programa presenta una serie de limitaciones que están ligadas a la inestabilidad de los vaivenes presupuestarios de cada gestión; el escaso desa- 
rrollo de la instancia participativa del programa y la limitación que presenta el hecho de que el programa no pueda constituirse en ley le otorga una inestabilidad dependiente de la gestión de turno (Fernández, 2020, p.3).

Estas limitaciones se inscriben en una estructura administrativa que, como han señalado estudios previos sobre Argentina y la región, cuentan con un grado de institucionalidad cultural baja o débil. Ello se refleja en la inestabilidad de los programas, la ausencia de documentación pública, la escasa planificación y sistematización de las acciones culturales, la inexperiencia en gestión cultural de quienes ocupan los cargos claves, entre otras debilidades (Rebón et al., 2013; Tasat et al., 2014; Mendes, 2015; Bayardo, 2016; Lerman, 2020). Desde una perspectiva histórica, también se ha señalado que tradicionalmente las políticas culturales en Argentina se caracterizaron por la falta de soporte presupuestario, la concentración de recursos en la Ciudad Autónoma de Buenos Aires y la inexistencia de una institucionalidad robusta (Zamorano, 2016).

En cuanto a las potencialidades del Programa, consideramos que éste habilitó la ayuda para sectores marginados o invisibilizados del campo cultural hegemónico, posibilitó flexibilizar procesos administrativos que facilitaron el acceso al Programa para muchas organizaciones, propuso una mirada de la política cultural que se distanció del paradigma de la democratización cultural (GarcíaCanclini, 1987) para promover proyectos genuinos de las comunidades que ya se encontraban en marcha, incluyó la dimensión territorial como constitutiva, propuso un espacio participativo a las organizaciones a partir de la conformación de un Consejo Comunitario, estableció instancias de encuentro regionales e internacionales y se mantuvo vigente a través de las gestiones gubernamentales de distinto signo político, incluyendo además iniciativas de capacitación (Fernández, 2020).

Siguiendo a Fraser (2000), podríamos decir que PdC se aproxima a una política redistributiva en lo económico en tanto realiza una transferencia efectiva de recursos a organizaciones culturales históricamente desplazadas de la esfera pública. Se trata, además, de una política de reconocimiento simbólico en tanto: a) detenta el "lenguaje del reconocimiento" (p.55), ya que es el mismo programa el que define cuáles son los puntos de la cultura comunitaria de Argentina (se denomina Puntos de Cultura a aquellas organizaciones que han ganado alguna convocatoria); bl implica una nueva jerarquización en la "representación” de las organizaciones culturales comunitarias a nivel institucional, es decir, un nuevo status en términos de "participación igualitaria" (p.63) ${ }^{6}$, y c) el hecho de que PdC sea una política presente en muchos países de la región, implica que las luchas a favor "de la redistribución y del reconocimiento" (Fraser, 2000) de estas organizaciones adquieren mayor resonancia al pasar de un marco de referencia local-nacional a uno global, volviendo legítimas las disputas en sus territorios, como discutiremos en los casos analizados.

\section{La Comunitaria}

La Cooperativa La Comunitaria es una organización social comunitaria con trece sedes en la provincia de Buenos Aires (Rooseveld, Sundblad, Mirapampa, Sansinena, González Moreno, Fortín Olavarría, San Mauricio, Trenque Lauquen, Colonia Seré, Villa Sauce Florentino Ameghino y Cañuelas) y 4 sedes en la provincia de La Pampa (General Pico, Realicó, Santa Isabel, Santa Rosa). Las actividades de la organización comenzaron en el 2006 de la mano de María Emilia De la Iglesia lactriz y comunicadora nacida en Sansinena), quien creó un grupo de teatro comunitario en su pueblo natal. Prontamente, las actividades del grupo se ampliaron geográficamente - a otros pueblos del Partido de Rivadavia y de la provincia de La Pampa- y se diversificaron hacia proyectos productivos, acciones sociales solidarias, talleres artísticos de distintas disciplinas y comedores. Su constitución como cooperativa (Matrícula Nacional No 46373) tuvo lugar en el año 2011, pero su mayor expansión se inició en el año 2017, momento en el cual comenzó a articular con otras organizaciones como el Movimiento de Trabajadores Excluidos en su rama Rural (MTE Rural) y y la Confederación de Trabajadores de la Economía Popular (CTEP)8.

Los referentes de La Comunitaria identifican en este momento un cambio de rumbo de la organización, en tanto reconfiguró su actividad hacia las necesidades básicas y profundas que los pueblos estaban atravesando: el desempleo, la precari- 
zación laboral, la pobreza. Dicha apertura y diversificación profundizó también el conflicto con el Municipio, el cual se agudizó hacia finales del año 2019 cuando el poder municipal no reconoció el contrato de comodato que había firmado con $L a$ Comunitaria. Allí se establecía que hasta diciembre de 2019 - con una renovación automática por cuatro años más en el caso de ser reelegido el Intendente Javier Reynoso-, La Comunitaria tendría la posesión en comodato del galpón que la organización utilizaba en la ciudad de América, Partido de Rivadavia. Al ser reelecto, el Intendente Reynoso comenzó una batalla legal y política que involucró a todo el pueblo, ya que desde el Municipio querían desalojar a la cooperativa de este galpón. Se trata de un espacio que se encontraba abandonado, fue ocupado por La Comunitaria en el año 2012 y reconstruido en su totalidad con la ayuda solidaria de los integrantes del grupo y vecinos de la zona. Su adquisición y puesta a punto constituye no solo un logro material sino también simbólico y político para la cooperativa. Aunque no discutiremos en profundidad este conflicto, sirve para ilustrar la complejidad del caso y la trayectoria del enfrentamiento con las autoridades municipales.

\subsection{La Comunitaria y Puntos}

La cooperativa La Comunitaria comenzó a participar del Movimiento de CVC y del colectivo Pueblo Hace Cultura en el 2012. En ese sentido, la organización, a través de sus referentes, fue parte de los debates por la formulación del programa y su diseño junto a otras organizaciones y representantes estatales.

\footnotetext{
Creo que el programa no solo tiene que apoyar a las organizaciones con apoyo económico sino que tiene que alentar la politización de la puesta en red más allá del programa, porque la idea de la cultura viva comunitaria es más que un programa, somos un movimiento. El programa puede ayudar, pero la perspectiva política tiene que seguir estando en las organizaciones. (Carla, referente de La Comunitaria, nov. 2019).
}

En relación a su incorporación como $\mathrm{PdC}$, la organización fue seleccionada en el 2016 por primera vez para ser beneficiaria del Programa. En esa oportunidad, se le brindó una ayuda de 50 mil pesos que se destinó a la compra de equipamien- to. Ese mismo año La Comunitaria participó del Encuentro Nacional de PdC que se realizó en la Ciudad de Buenos Aires y De la Iglesia fue elegida como la representante de la Provincia de Buenos Aires en el Consejo Cultural Comunitario. En el año 2017 recibió también financiación de PdC para la organización del Encuentro de Redes de CVC que se desarrolló en Rivadavia, como antesala del Primer Congreso Nacional de CVC (2019). En esa ocasión el Programa PdC brindó ayuda en la compra de un almuerzo comunitario y algunos pasajes. Para el mismo evento La Comunitaria obtuvo el apoyo del Programa de Ibercultura Viva.

En el contexto del 2016, 2017 fue importante el apoyo del programa $(\mathrm{PdC})$ porque acá teníamos una situación super adversa y no se podía creer que ese programa nos daba apoyo y el Municipio no nos daba ni agua. En ese sentido, fue muy importante. (Carla, referente de La Comunitaria, 11/2019).

Así, el vínculo entre La Comunitaria y PdC ha fortalecido mayormente la dimensión institucional de la organización, en tanto su impacto estuvo más vinculado a la generación de lazos, la búsqueda de financiación -que, si bien fue significativa para el grupo, no fue determinante- y la posibilidad de intervenir en instancias deliberativas en relación al Programa y su implementación, que con acciones en territorio.

Un informe elaborado por el equipo de PdC y distintas universidades nacionales (Ministerio de Cultura de la Nación, 2015), destaca ciertos puntos de partida interesantes para pensar nuestro caso. $\mathrm{Si}$ bien dicho informe no considera entre los casos que estudió el de La Comunitaria (porque no ingresó al programa hasta el 2016), sí especifica la idea de que las organizaciones comunitarias son las protagonistas de las transformaciones de sus territorios, porque sus integrantes conocen profundamente la matriz cultural de sus comunidades, sus problemas y necesidades. En ese sentido, los recursos que brinda el Estado ly cuya distribución y jerarquización merece una discusión aparte) deben ser gestionados por las organizaciones en función del desarrollo de su trabajo territorial. Por otro lado, se remarca como requisito la recuperación de los saberes locales, en una revalorización de las estrategias de la economía popular, y la dimensión colectiva de articulación entre instituciones y espacios comunitarios locales con el Estado. 
A partir de estas premisas esbozadas por informe, nos preguntamos cuál es la injerencia de $\mathrm{PdC}$ en la dimensión territorial del desarrollo de La Comunitaria, entendiendo por la misma las transformaciones concretas que se dan en el espacio geográfico en sentido amplio (Brizuela \& Barros, 2017) -como la toma de espacios, la modificación edilicia, la creación de nuevas sedes, la ampliación de actividades, la promoción de instancias de reflexión en las comunidades, la transformación o creación de normativas, la articulación con otras organizaciones e instituciones-, tanto como aquellas que afectan las subjetividades de sus integrantes y que remarcaban Barbieri, Partal y Merino (2011) - la identidad, participación ciudadana, el reconocimiento y gestión del conflicto, la revalorización de las "clases no productivas" (p. 487), el desarrollo autónomo y promoción de la creatividad de las personas, entre otras-.

Consideramos que el programa $\mathrm{PdC}$ no ha tenido una injerencia significativa en la dimensión territorial de La Comunitaria, ya que esta organización posee gran independencia de acción en la proyección de actividades, las cuales no se planifican en base a los recursos disponibles o a las posibles ayudas económicas, sino que se ejecutan a partir de estrategias múltiples, dentro de las cuales los lazos de solidaridad y asociativismo son cruciales. Por otro lado, La Comunitaria ha ido incorporando con el paso de los años una mayor articulación con una multiplicidad de instituciones y organizaciones, lo cual alentó la diversificación en las estrategias de acción (Fernández, 2020). En ese sentido, las acciones de La Comunitaria que generaron instancias de transformación más evidentes en el territorio no se iniciaron ni estuvieron condicionadas por los beneficios del programa PdC.

En el caso de La Comunitaria observamos que se trata de una organización social que se ha vinculado fuertemente con políticas públicas culturales y sociales, pero su mayor trabajo territorial está estructurado sobre un entramado asociativo que involucra tanto a las comunidades en las cuales están ubicadas sus sedes, como a distintas organizaciones de base que trabajan con sectores productivos populares rurales en distintas partes del país.

El programa PdC fue importante para nosotros. A La Comunitaria no le cambió la vida, sino que le cambió la vida ser parte del movimiento de cultura viva comunitaria, articular con las otras organizaciones y todo lo que vino después. (Carla, referente de La Comunitaria, Nov. 2019).

Tal como vemos, para el caso de La Comunitaria el programa $\mathrm{PdC}$ ha sido relevante en tanto política de ingreso a una multiplicidad de espacios de intercambio y reconocimiento con otras organizaciones de la cultura comunitaria, así como también abrió las puertas al diálogo estrecho con referentes territoriales del ámbito estatal. La dimensión institucional, tal como la llamamos, es parte fundamental de la proyección de la organización, tanto dentro de su región como también en un sentido amplio, a nivel nacional y latinoamericano. Este aspecto, profundamente legitimador, unido a la relevancia que adquirió el trabajo territorial de La Comunitaria en sus 17 sedes, configuran una de las mayores fortalezas de la organización en la actualidad.

\section{Chacras para Todos}

Chacras para Todos es un proyecto artístico comunitario ubicado en la localidad de Chacras de Coria del departamento de Luján de Cuyo en la provincia de Mendoza (Argentina). Surgió en el año 2008 a partir de la iniciativa de tres residentes del lugar que percibían a la comunidad como fragmentada tras experimentar durante los últimos años un crecimiento demográfico acelerado de su población y la transformación del lugar en un polo residencial y gastronómico de los sectores con mayor poder adquisitivo de la provincia. El objetivo principal de Chacras para Todos fue trabajar a favor de la integración en la diversidad y recuperar la identidad local "perdida" (CHPT, 2017, s/n)", conformándose, en un comienzo, como un "proyecto artístico comunitario independiente", que abarcaba un amplio abanico de actividades y disciplinas artísticas. Debido a su capacidad de gestión y de articulación con otras organizaciones, instituciones y redes, uno de los aspectos más desarrollados de Chacras para Todos ha sido el institucional. Así, además de las actividades de formación, ensayos y presentaciones artísticas, han dinamizado la vida artístico-cultural de su comunidad a partir de la organización de festejos tradicionales como el carnaval y las vendimias distritales. Este aspecto también se vio fortalecido por la capacidad de acceder a convocatorias y apoyos 
financieros gracias al perfil profesional del equipo coordinador y de muchos/as integrantes.

Debido a esta trayectoria, Chacras para Todos ha cumplido un rol protagónico en la conformación de las redes y movimientos locales y regionales, así como ha disputado espacios de reconocimiento de la cultura comunitaria en la esfera pública (Sánchez-Salinas, 2020). Se trata de una organización que se transformó en un puente entre los distintos ámbitos de lo local (público, privado, comunitario) y en los distintos niveles (municipal, provincial y nacional) dentro del sector cultural comunitario

\subsection{De Punto a Círculo de Cultura: la extensión a nuevos horizontes}

El primer intercambio de la organización con PdC fue en el año 2013 cuando lanzaron una convocatoria que estimulaba la expansión de actividades culturales a partir de proyectos ya existentes y con antigüedad en un mismo territorio. Era una línea de apoyo destinada a grandes centros culturales comunitarios: los "Círculos de Cultura"10, una categoría del Programa en articulación con el Plan Nacional de Igualdad Cultural ${ }^{11}$. El objetivo era fortalecer proyectos culturales comunitarios de todo el país para mejorar su funcionamiento y estimularlos a replicar su experiencia en la formación de emprendimientos cooperativos con el fin de desarrollar talleres, espectáculos barriales, imprentas populares, discográficas, radios o canales de televisión comunitarios. La convocatoria estipulaba que cada Círculo de Cultura debía conformar tres cooperativas culturales en su entorno, debiendo estar inscritas en el Instituto Nacional de Asociativismo y Economía Social (INAES).

Chacras para Todos resultó seleccionado con el proyecto "De acequias, arte y memoria, entre todos hacemos historia" (CHPT, 2013), convirtiéndose en el primer y único "Círculo de Cultura" de la Región Nuevo Cuyo (de 24 proyectos presentados, se seleccionaron siete, uno por región). Fue así que, en el año 2013, la organización se enfrentó al desafío de emprender actividades fuera del territorio y al compromiso de que esas iniciativas se constituyeran como una cooperativa cultural ${ }^{\mathbf{1 2}}$. En consecuencia, destacamos que, para el caso estudiado, el vínculo con el Programa implicó una amplia- ción efectiva del alcance de su proyecto. Incluso la organización pudo contribuir con la formación no sólo de tres cooperativas con financiamiento del programa, sino que también ayudó a que surgieran cuatro más sin financiamiento. Este énfasis en lo territorial por parte de PdC puede enmarcarse en un proceso más amplio de "territorialización de dispositivos institucionales" que caracterizó la política institucional de Néstor Kirchner y Cristina Fernández de Kirchner (Perelmiter, 2016, p.222). Dentro de este esquema, la política de PdC a partir del desarrollo de modalidades que complementaban la mera transferencia de recursos, logró una mayor proximidad a los "territorios" a través de las organizaciones de la cultura comunitaria. Para nuestro caso, este proceso de ampliación territorial y promoción cultural a partir de la conformación de cooperativas, posicionó a Chacras para Todos como un referente en términos de gestión y formalización de actividades artísticas y culturales que años más tarde le permitiría al grupo volver legítima una disputa con el gobierno municipal por el uso del teatro Leonardo Favio ubicado frente a la plaza principal de Chacras de Coria (Luján de Cuyo, Mendoza).

Si bien por el alcance de sus actividades Chacras para Todos siempre había sido un actor relevante como productor de políticas culturales locales, esto se hizo manifiesto cuando entró en disputa con el gobierno local intentando "resignificar las interpretaciones dominantes de lo político o desafiando prácticas políticas establecidas" (Álvarez, Dagnino \& Escobar, 2008, p. 24).

\subsection{Usos y apropiaciones estratégicas de PdC}

Durante los primeros cinco años (2008-2012), Chacras para Todos "había itinerado por diferentes instituciones lescuelas, espacios del municipio, la parroquia), hasta que lograron recuperar el antiguo cine-teatro de la localidad ubicado frente a la plaza principal que había permanecido cerrado la última década" (Sánchez Salinas, 2020, p. 163). Después de ocho años de mantener un pacto para el uso del espacio con el municipio local, en 2016 les solicitaron retirarse argumentando que realizarían una modernización del teatro. Ante esta situación, Chacras para Todos dinamizó la conformación de una Red local (La Red Cultural de Chacras de Coria), reuniendo a un número significativo de instituciones y agentes culturales de la localidad en defensa de 
una propuesta de co-gestión del teatro (municipio y la Red) ${ }^{13}$.

En este proceso, Chacras para Todos fue involucrando a los integrantes de la Red en disputas y sentidos que circulaban en el movimiento de CVC. La estrategia fue movilizar repertorios de actores no locales que legitimaran el accionar del grupo en otras escalas, apoyándose, a su vez, en el valor que le otorgaba ser una referencia central del Programa en tanto único Círculo de Cultura de la región Cuyo. Fue así que lograron visibilizar el conflicto local, consiguieron apoyos para defender la causa del teatro e, incluso, gestionaron subsidios para solventar sus proyectos. En este sentido, el evento más significativo fue el Aniversario de la Red Cultural de Chacras de Coria (2017), para el cual ganaron un subsidio internacional del programa Ibercultura Viva en la convocatoria a Redes. El festejo se realizó en co-gestión con PdC en el marco del Encuentro Regional de Puntos de Cultura de Nuevo Cuyo. Esta alianza le brindó a la Red fondos para pasajes, alojamiento y comida para contar con un mayor número de organizaciones de otros departamentos y provincias. También al Programa le permitió un anclaje local para difundir y fortalecer el Consejo de Cultura Comunitaria en una región donde la política no lograba el alcance esperado.

Es probable que, sin el apoyo del movimiento de CVC, el aporte financiero de Ibercultura Viva y la legitimidad de Chacras para Todos en tanto "Círculo de Cultura", la posibilidad de crear y sostener la red local hubiese estado debilitada. Y, a la inversa, solamente con el apoyo local no hubiera alcanzado para darle visibilidad al conflicto y sostener el derecho a la co-gestión del espacio. En este juego de escalas, entre lo local-municipal, lo nacional y lo internacional, quedó de manifiesto que el hecho de ser reconocidos por $\mathrm{PdC}$ fue clave en el proceso de volver legítima la disputa por el espacio en y más allá de la comunidad de Chacras.

En síntesis, Chacras para Todos apeló a una estrategia local (la conformación de la Red Cultural de Chacras) a partir de un repertorio de un actor social nacional (el programa $\mathrm{PdC}$ ) y trasnacional (el movimiento de CVC) para legitimar sus concepciones. El hecho de haber dejado de responder a referentes territoriales y empezar a estar crecientemente influenciado por procesos de otros espacios geográficos, no implicó que aquella dis- puta resultara des-territorializada, en todo caso fue "re-territorializada", "trans-territorializada" o “multiterritorializada" (Mato, 2007). La estrategia "multiterritorial" le permitió legitimar la disputa con el municipio no sólo por la propuesta de cogestión de un espacio cultural central en esa comunidad, sino también por el modelo de gestión de las políticas culturales, donde lo que estaba en juego era el grado de apertura del Estado para el diseño de las políticas locales. En definitiva, después de diez años de trabajo, lo que el conflicto por la reforma del teatro evidenció fue que Chacras para Todos era un actor legítimo para disputar la orientación de las políticas culturales en el territorio y que el programa de $\mathrm{PdC}$ y las redes culturales jugaron un rol decisivo en dicho proceso de legitimación.

\section{Conclusiones}

A partir de lo analizado, podemos afirmar que PdC es una política de alto impacto a nivel simbólico que incide en la dimensión institucional de las organizaciones, volviendo legítimo el accionar de éstas en sus comunidades. En el caso de Chacras para todos, el Programa promovió que el grupo avance en la constitución de un proyecto cultural más amplio y territorialmente extendido, lo cual lo fortaleció institucionalmente y configuró un rasgo legitimador tanto a nivel nacional como regional. Las alianzas establecidas con distintas redes y el Programa resultaron estratégicas para que la organización visibilice y se posicione ante el conflicto con el municipio local. En el caso de La Comunitaria, se trata de una organización que contaba con un entramado asociativo fuerte previo a su contacto con el Programa. En ese sentido, la injerencia de PdC a nivel territorial no fue significativa, pero sí lo fue en la dimensión institucional, al igual que el caso de Chacras. Es notorio que en ambos casos la vinculación con $\mathrm{PdC}$ ha sido relevante, pero fueron las organizaciones las encargadas de canalizar, gestionar y desarrollar sus proyectos culturales.

Otro aspecto significativo que se desprende del análisis señala la divergencia en los usos y apropiaciones que cada organización realiza del Programa, en función de los objetivos que plantean sus proyectos político-culturales, el rol de sus líderes y su situación coyuntural (especialmente su anclaje territorial). La indagación muestra que distintos usos 
y apropiaciones de PdC se vuelven una estrategia eficaz en la gestión de los conflictos locales, cuando hay divergencia de modelos, criterios o afinidades políticas entre los gobiernos municipales y las organizaciones.

Por último, destacamos que, para el caso argentino, y pese a la débil institucionalidad de los organismos de cultura, el Programa ha tenido mayor impacto como política de reconocimiento que como política pública de transferencia de recursos financieros, como apuntan también otros trabajos (Sánchez-Salinas, 2020). Si bien siguen siendo múltiples las limitaciones del Estado (burocráticas, administrativas y culturales) al momento de garantizar instancias participativas en la gestión de las políticas culturales públicas -y, en este sentido, los objetivos principales de PdC encuentran un problema estructural (Turino, 2013), el análisis muestra que el Programa alcanza de forma gradual e incipiente la dimensión política de la representación y la dimensión cultural del reconocimiento (Fraser, 2006). Señalamos la importancia de continuar estudiando este tipo de políticas que trabajan con organizaciones vinculadas a comunidades e identidades históricamente desplazadas y excluidas, dado que su permanencia garantiza el ingreso efectivo de las mismas dentro de la estructura gubernamental y nos acerca al horizonte de políticas culturales más igualitarias.

\section{Notas}

1. En el siguiente apartado $\left(\mathrm{n}^{\circ} 2\right)$ se detallan y describen los antecedentes.

2. El teatro comunitario argentino es un teatro "de vecinos para vecinos", realizado por personas no profesionales del teatro (con excepción del director o directora y eventualmente miembros del equipo de coordinación), que se reúnen para elaborar mediante el ejercicio de memoria colectiva una obra de teatro que cuente la historia de su ciudad, pueblo o barrio. Los grupos son heterogéneos tanto en su constitución etaria como en la condición socioeconómica, política y religiosa de sus integrantes. Son grupos autogestivos que suelen utilizar el espacio público para sus puestas en escena. En Argentina existen aproximadamente 50 grupos.

3. Los entrevistados serán presentados a través de seudónimos.

4. La Red Nacional de Teatro Comunitario fue creada en 2003 como una instancia de organización colectiva e intercambio para los grupos del país. En Argen- tina tuvo un rol protagónico en las disputas con el Estado por el reconocimiento de la actividad teatral comunitaria y en Latinoamérica en la conformación de espacios como la Red Latinoamericana de Arte y Transformación Social y la Plataforma Puente Cultura Viva Comunitaria.

5. El Consejo Cultural Comunitario fue una plataforma creada por el Programa en el Encuentro Nacional de PdC 2016, en la que participaban representantes de organizaciones, redes y colectivos culturales de todo el país. Además de estar constituido por representantes de todas las regiones, tenía participación de distintas redes temáticas dentro de las cuales se incluía la Red Nacional de Teatro Comunitario donde participaban La Comunitaria y Chacras para Todos. Actualmente no está en funcionamiento.

6. Para la autora la política del reconocimiento no se detiene en el modelo de la identidad, sino que "aspira a generar soluciones institucionales" (Fraser, 2000, p.64). Dentro del problema del marco de la justicia, incluye tres dimensiones: “la dimensión política de la representación, la dimensión económica de la distribución y la dimensión cultural del reconocimiento" (Fraser, 2006, p.35).

7. El Movimiento de Trabajadores Excluidos es una organización social nacional que impulsó la conformación de cooperativas y grupos de economía popular que luchan por la demanda de tierra, techo y trabajo. Este movimiento tiene una rama rural denominada MTE Rural.

8. La Confederación de Trabajadores de la Economía Popular (CTEP) es una organización gremial independiente de todos los partidos políticos, representativa de los trabajadores de la economía popular y sus familias.

9. La abreviación del nombre del grupo "CHPT" seguida de fecha, indica la fuente del grupo consultada (proyectos de subsidios, publicaciones en redes sociales, notas, entre otras) y se detallan en las referencias al final del artículo.

10. Del mismo modo que PdC había tomado como referencia el programa homónimo de Brasil, la idea de los Círculos de Cultura se asemejaba a los Pontões de Cultura de la política brasileña.

11. El Plan Nacional de Igualdad Cultural "Inclusión en la diversidad" fue una política implementada por el Ministerio de Planificación Federal, Inversión Pública y Servicios, y la Secretaría de Cultura de Presidencia de la Nación en el año 2012. La articulación con el Programa PdC se realizó a través del Programa de Fondos Concursables del Plan.

12. Para profundizar sobre las implicancias para la organización de la extensión de sus actividades a nuevos territorios cf. Sánchez Salinas (2018b, 2020).

13. La propuesta de la Red Cultural de Chacras era la co-gestión del teatro (gobierno y organizaciones), así como la participación en el diseño del proyecto de modernización del espacio. Para ampliar sobre la disputa cf. Sánchez-Salinas (2018b). 


\section{Referencias}

Álvarez, S., Escobar, A. \& Dagnino, E. (2008). “Lo cultural y lo político en los movimientos sociales de América latina". En Culturas en América Latina y el Perú. Luchas, estudios críticos y experiencias. III Foro de democratización global: Culturas para la transformación social. Lima, Perú.

Barbieri, N., Partal, A. \& Merino, E. (2011). Nuevas políticas, nuevas miradas y metodologías de evaluación. ¿Cómo evaluar el retorno social de las políticas culturales?. Papers, Revista de Sociología, 96, 477-500. https://doi.org/10.5565/rev/papers/v96n2.139

Barbosa, F. \& Calabre, L. (2011) (Orgs). Pontos de Cultura: olhares sobre o programa cultura viva. IPEA.

Bayardo, R. (2016). Hacia un panorama del desarrollo de la gestión cultural en Argentina. En A. Canelas Rubim, C. Yañez Canal \& R. Bayardo. (Org.), Panorama da gestão cultural na Ibero-américa (n²3, pp.15-34). EDUFBA.

Benhabib, D. (2018). “Puntos de Cultura. Dinámica de lo impensado”. En V. Prato \& S. Segura (Ed), Estado, sociedad civil y políticas culturales. Rupturas y continuidades en Argentina entre 2003 y 2017 (pp. 231-256). RGC Ediciones.

Bentes, I. (2016). "Cultura viva: Proceso culturais innovadores". En M. Ortiz (Comp), Cultura urbana para la inclusión en América Latina. SEGIB Secretaría General Iberoamericana.

Bordat-Chauvin, E. (2020). La cultura y la austeridad. Análisis etnográfico en un programa cultural argentino. Alteridades, 30(60), 67-76. www.doi.org/10.24275/uam/izt/dcsh/ alt/2020v30n60/Bordat

Brizuela, J. \& Barros, J.M. (2017, 12-15 de septiembre). Dimensões de uma política trasnacional intercultural: o Programa Puntos de Cultura na Argentina. [Presentación de Ponencia] XIII Encontro de estudos multidisciplinares em cultura, Salvador, Bahía, Brasil.

Chacras para Todos (2013) "De acequias, arte y memoria, entre todos hacemos historia”. (Proyecto de subsidio presentado en convocatoria de Círculos de Cultura 2013 del Programa, inédito).

Chacras para Todos. (2017). Rendición de subsidio Circulación Internacional de Elencos del Instituto Nacional del Teatro, obtenido para la gira de la obra "De muros a puentes". (Inédito).

Fernández, C. (2015) La potencia en la escena. Teatro Comunitario de Rivadavia : Historicidad, política, actores y sujetos en juego/s (2010-2014) (Tesis de posgrado presentada en la Universidad Nacional de La Plata. Facultad de Humanidades y Ciencias de la Educación para optar al grado de Doctora en Ciencias Sociales). https://www.memoria.fahce. unlp.edu.ar/tesis/te.1170/te.1170.pdf

Fernández, C., Bidegain, M., Falzari, G. \& Sánchez-Salinas, R. (2017). “Nuevas estrategias colectivas de gestión teatral. El caso del grupo de Teatro Comunitario de Rivadavia" (pp.140195). En Modelos de Gestión Teatral, Casos y experiencias 1. Colección Estudios Teatrales. Ediciones del Instituto Nacional del Teatro. https://www.recursosculturales.com/ descarga-gratis-modelos-de-gestion-teatral-trabajo-publicado-por-el-int/

Fernández, C. (2018). Políticas culturales en acto. Teatro comunitario argentino: entre el Estado y la autogestión. Papers, Revista de Sociología, (3), 447-477. https://doi.org/10.5565/rev/ papers. 2430

Fernández, C. (2020). Estado y políticas culturales en Argentina. Un análisis comparativo entre el Kirchnerismo y la Alianza Cambiemos (2007-2017). Sociohistórica, (45), 1-17. https:// doi.org/10.24215/18521606e102

Fraser, N. (2000) Nuevas reflexiones sobre el reconocimiento. New Left Review, 3, 55-68. https:// newleftreview.es/issues/4/articles/nancy-fraser-nuevas-reflexiones-sobre-el-reconocimiento.pdf

Fraser, N. (2006) Reinventar la justicia en un mundo globalizado. New Left Review, 36, 31-50. https://newleftreview.es/issues/36/articles/nancy-fraser-reinventar-la-justicia-enun-mundo-globalizado.pdf

Fuentes-Firmani, E. (s/f). Programa Puntos de Cultura de la Secretaría de Cultura de Presidencia de la Nación. Una propuesta de mejoramiento de sus resultados (Tesina de grado inédita para la Licenciatura en Gestión del Arte y la Cultural. Universidad Nacional de Tres de Febrero, Tres de Febrero, Argentina.

García-Canclini, N. (Ed.) (1987). Políticas culturales en América Latina. Grijalbo.

Lacarrieu, M. \& Cerdeira, M. (2016). “Institucionalidad y políticas culturales en Argentina. Límites y tensiones de los paradigmas de democratización y democracia cultural". Políticas Culturales, 9,10-33. https://ri.conicet.gov.ar/bitstream/handle/11336/71896/CONICET_Digital_Nro.787bc26b-a3c8-42b0-8111-524e28e2d490_A.pdf?sequence=2\&isAllowe $\bar{d}=y$ 
Lerman, G. (2020). La comunidad futura. Ruinas, instituciones culturales y otras imaginerías. RGC Libros.

Lucesole, R. (2016). Puntos de Cultura. Política cultural y construcción de subjetividades. Reflexiones sobre la práctica (2011 - 2015). (Trabajo Integral final de la Especialización en Gestión Cultural y Políticas Culturales, Instituto de Altos Estudios Sociales). Universidad Nacional de San Martín, Argentina. https://ri.unsam.edu.ar/handle/123456789/573

Marradi A., Archenti N., \& Piovani J.I. (2007). Metodología de las ciencias sociales. Emecé.

Mato, D. (2007). “Cultura, Comunicación y Transformaciones sociales en tiempos de globalización. Perspectivas latinoamericanas". En D. Mato, \& F. Maldonado (Eds.), Cultura y Transformaciones sociales en tiempos de globalización (pp.10-75). Perspectivas latinoamericanas.CLACSO.

Mello, D. (2018). Políticas culturales en disputa. Un estudio antropológico de los sentidos de cultura, cultura popular y política cultural en Puntos de Cultura. (Tesis de Licenciatura en Ciencias Antropológicas). Universidad de Buenos Aires, Buenos Aires, Argentina. http://iberculturaviva.org/wp-content/uploads/2020/03/Politicas_culturales_en_disputa_Un_estud.pdf

Mello, D. (2019). El programa Puntos de cultura y sus destinatarios: un estudio de caso en la Provincia de Buenos Aires. Katálisis,1,181-190. https://doi.org/10.1590/1982$02592019 v 22 n 1 p 181$

Mendes, P. (2015) Políticas Culturales: Rumbo y Deriva. Estudio de casos de la (ex) Secretaría de Cultura de la Nación. RGC Libros.

Mihal, I. (2014). Inclusión digital y gestión cultural en el Mercosur: el Programa Puntos de Cultura. Estudios de Comunicación y Política, 34, 126-137. http://sgpwe.izt.uam.mx/files/users/ uami/ana/IvanaMihalPuntosCultura.pdf

Ministerio de Cultura de la Nación. (2015). Puntos de Cultura, una política transformadora. Relevamiento y análisis del impacto territorial. Recuperado de: https://puntos.cultura.gob.ar/ wp-content/uploads/sites/17/2018/08/Puntos-de-cultura_Maquetacio\%CC\%81n-1.pdf

Noel, G. (2011). Guardianes del Paraíso. Génesis y Genealogía de una Identidad Colectiva en Mar de las Pampas, Provincia de Buenos Aires. Revista del Museo de Antropología, 4 (1), 211 226. https://doi.org/10.31048/1852.4826.v4.n1.5487

Organización de las Naciones Unidas para la Educación, la Ciencia y la Cultura. (2001). Declaración Universal de la UNESCO sobre la Diversidad Cultural. Recuperado de: http://portal. unesco.org/es/ev.php-URL_ID=13179\&URL_DO=DO_TOPIC\&URL_SECTION=201.html

Organización de las Naciones Unidas para la Educación, la Ciencia y la Cultura. (2005). Convención sobre la protección y la promoción de la diversidad de las expresiones culturales. Recuperado de: http://www.unesco.org/new/es/culture/themes/cultural-diversity/culturalexpressions/the-convention/convention-text

Perelmiter, L. (2016). Burocracia plebeya. La trastienda de la asistencia social en el Estado Argentino. UNSAM EDITA.

Prato, A. V. \& Segura, M. S. (Eds.). Estado, sociedad civil y políticas culturales. Rupturas y continuidades entre 2003 y 2017. RGC Ediciones.

Rachid. S. (2016). Cultura Viva: Enrendando ciudadanía en Bahía. (Trabajo final integrador Especialización en Gestión Cultural, Instituto de Altos Estudios Sociales, Universidad Nacional de San Martín). https://ri.unsam.edu.ar/handle/123456789/168

Rebon, M., Tasat, J., Mendes Calado, P., Basualdo, J., Martíntez, S. \& Fiore, L. (2013). “La cultura como política pública: la gestión de la cultura a nivel local" (197-204). En Indicadores Culturales: cuadernos de políticas culturales. EDUNTREF.

Secretaría de Cultura de la Nación. (2011). Resolución 2641/2011. Creación del Programa Nacional Puntos de Cultura. Argentina.

Rodriguez De Melo, B. E. (2016). Desenvolvimento e políticas culturais de base comunitária na América do Sul: estudo comparado Brasil-Argentina. (Tesis para Mestrado em Desenvolvimento Sociedade e Cooperação Internacional). Universidade de Brasília, Brasília, Brasil. https://repositorio.unb.br/handle/10482/20771

Sánchez-Salinas, R. (2020). Las políticas culturales y su rol en la definición de elementos identitarios en organizaciones comunitarias: el caso de Chacras para Todos en la provincia de Mendoza (Argentina). Cuaderno 116 | Centro de Estudios en Diseño y Comunicación, 159 - 178. https://doi.org/10.18682/cdc.vi116.4139

Sánchez-Salinas, R. (2018a). Resonancias actuales de la comunidad: el teatro comunitario argentino como espacio de recreación de lazos de pertenencia. Revista Question, (59), 1-18. https://doi.org/10.24215/16696581e067 
Sánchez-Salinas, R. (2018b). Las organizaciones culturales y su vínculo con el Estado: el caso del teatro comunitario mendocino. En M. Segura \& A. Prato (Eds.) Estado, sociedad civil y políticas culturales. Rupturas y continuidades entre 2003 y 2017. RCG Libros.

Sánchez-Salinas, R. (Coord.) (2014). El movimiento teatral comunitario argentino - Reflexiones acerca de la experiencia en la última década (2001-2011). Ediciones del CCC.

Santini, A. (2017). Cultura viva comunitária. Políticas culturais no Brasil e na América Latina. ANF Produções.

Tasat, J. (Comp.) (2014). Políticas culturales públicas. EDUNTREF.

Turino, C. (2013). Puntos de cultura. Cultura Viva en Movimiento. RGC Ediciones.

Vilutis, L. (2009). Cultura e juventude: a formação dos jovens nos pontos de cultura. (DissertaçãoMestrado, Faculdade de Educação da Universidade: Programa de Pós-Graduação em Educação. Área de Concentraçã́o: Cultura, Organização e Educação). https://www.teses.usp.br/teses/disponiveis/48/48134/tde-23092009-132908/en.php

Wajnerman, C. (2018). “Interculturalidad y políticas públicas: Estado y organizaciones en el programa Puntos de Cultura". En A. Prato \& M. Segura (Eds), Estado, sociedad civil y políticas culturales. Rupturas y continuidades en Argentina entre 2003 y 2007 (pp.257-278). RGC Ediciones.

Wortman, A. (2017). Políticas culturales y legitimidad política en tiempos de crisis. El caso del Programa Puntos de Cultura de Argentina. Revista Pol. Cult. 1, 138-160. https://www. researchgate.net/publication/324955387_Politicas_culturales_y_legitimidad_politica_ en_tiempos_de_crisis_el_caso_del_Programa_Puntos_de_Cultura_en_Argentina.

Zamorano, M. (2016). La transformación de las políticas culturales en Argentina durante la primera década kirchnerista: entre la hegemonía y la diversidad. Aposta. Revista de Ciencias Sociales, 70, 53-83. http://apostadigital.com/revistav3/hemeroteca/zamorano1.pdf>

\section{- Sobre las autoras:}

Romina Sánchez Salinas es Doctora en Sociología (IDAES-UNSAM), Licenciada en Sociología (UNCuyo Mendoza) y especialista en Gestión y Política en Comunicación y Cultura (FLACSO Argentina). Realiza estudios post-doctorales con beca CONICET con sede en el Instituto Multidisciplinario de Estudios Sociales Contemporáneos de la Facultad de Filosofía y Letras de la UNCuyo. Profesora en la Universidad Nacional de Tres de Febrero. Ha realizado distintos estudios e informes en el ámbito público siendo parte de áreas estadísticas de cultura (Instituto Nacional del Teatro y en Data Cultura del Ministerio de Cultura del GCBA). Es miembro del Observatorio de Políticas Culturales y Culturas Políticas del Centro Cultural de la Cooperación.

Clarisa Fernández es Doctora y Magister en Ciencias Sociales (FaHCE, UNLP), Especialista en Producción de textos críticos y Difusión mediática de las artes (UNA), Licenciada y Profesora en Comunicación Social (FPyCS, UNLP). Es Investigadora Asistente del CONICET y docente en la materia Política de la Información de la Carrera de Bibliotecología de la FaHCE, UNLP. Trabaja con temáticas vinculadas a las políticas públicas culturales y organizaciones artísticas comunitarias, específicamente grupos de teatro comunitario argentino

\section{- ¿Cómo citar?}

Sánchez Salinas, R., \& Fernández, C. (2021). La política cultural en territorio: análisis de Puntos de Cultura desde la dimensión del reconocimiento. Comunicación y Medios. (44), 106-117. https://doi.org/110.5354/0719-1529.2021.60975 